\title{
Why are people with asthma more susceptible to influenza?
}

\author{
Peter A.B. Wark (i) \\ Affiliation: Centre for Healthy Lungs, Hunter Medical Research Institute, University of Newcastle, New \\ Lambton, Australia. \\ Correspondence: Peter A.B. Wark, University of Newcastle, Centre for Asthma and Respiratory Disease, Dept \\ of Respiratory and Sleep Medicine, John Hunter Hospital, Lookout Rd, New Lambton, New South Wales, 2305, \\ Australia. E-mail: Peter.warkanewcastle.edu.au
}

@ERSpublications

The effect of viruses such as influenza on asthma exacerbations is not the result of either exaggerated type 1 or 2 immune responses in those with asthma. http://bit.ly/2maJHVV

Cite this article as: Wark PAB. Why are people with asthma more susceptible to influenza? Eur Respir J 2019; 54: 1901748 [https://doi.org/10.1183/13993003.01748-2019].

In 1860, Henry Hyde Salter characterised the important association between virus infection and acute asthma. He understood viruses caused severe exacerbations: "The asthma consequent on cold on the chest (bronchitis) is of a most painful and distressing kind; unlike that produced by cold directly, it often lasts for days." He also realised that viral infections worsened airway inflammation and bronchial hyperresponsiveness: "I myself have no doubt that in ordinary acute bronchitis a good deal of the dyspnoea ... is due not only to the mucous membrane being tumid from inflammation and so narrowing the calibre of the tubes, but to active contraction from the irritation and exalted sensibility that the inflammation gives rise to" [1]. While an appreciation of the importance of airway inflammation in asthma evolved throughout the 1980s, molecular diagnostics were required to prove the dominant role virus infection played as the trigger of acute asthma [2]. Virus infections have been consistently demonstrated as the most prevalent trigger of acute severe asthma, for children and adults [3]. This susceptibility was demonstrated during the influenza pandemic of 2009 , where asthma was repeatedly demonstrated as one of the most prevalent risk factors for hospitalisation $[4,5]$. The reason asthmatics are susceptible to the effects of virus infections, though, remains elusive and our understanding is confounded by a number of paradoxes.

Asthma as a cause of impaired antiviral immunity is an obvious place to start. In 2005 it was first demonstrated that asthmatic airway epithelial cells responded to rhinovirus infection with impaired innate, interferon- $\beta$ responses [6]; although over the next 10 years this finding was not always replicated, the balance of studies suggested this impaired response was seen more often in those with poor asthma control and was only a feature of early impaired innate responses [7]. While cytokines such as transforming growth factor- $\beta$ [8] and interleukin (IL)-13 [9], released during asthmatic inflammation, were shown to impair these innate epithelial type I and III interferon responses to viruses, in vitro. Allergic inflammation was also implicated in impaired innate antiviral responses, with crosslinking of the high-affinity $\operatorname{IgE}$ receptor (FceRI) on plasmacytoid dendritic cells, associated with reduced expression of TLR-7 and interferon- $\alpha$ release in response to influenza [10]. These findings are supported by the clinical observations, that poor asthma control increases the risk of exacerbations, but it is here that a number of paradoxes emerge. First, the presence of eosinophilic/type 2 airway inflammation in animal models makes them more resistant to influenza $[11,12]$. The second is that in people, control of asthmatic inflammation 
involves the use of inhaled corticosteroids, while acute asthma is successfully treated by high dose systemic corticosteroids, both of which impair antiviral responses. This is not the case, in asthma where the use of systemic corticosteroids is protective [13]. While the opposite is true in those hospitalised with influenza without asthma [14].

The influenza pandemic of 2009 provided a unique opportunity, unlike seasonal influenza the "novel" virus did not target those at the extremes of age and its impact had the greatest effect on other, less appreciated, at risk populations; those with asthma, obesity and pregnancy [5]. The paper by JHA et al. [15] describes the effect of influenza H1N1pdm09 on 133 adults admitted during this time to UK hospitals. Asthma was the most prevalent pre-existent condition, in $30 \%$, with most being female. In asthma the disease was less severe, with fewer intubations and a shorter length of stay. Suboptimal prior asthma control could be inferred, with only $16 / 40$ asthmatics on regular inhaled corticosteroids. Uniquely the investigators studied the pathogenesis of this naturally occurring event, looking at immune responses within $24 \mathrm{~h}$ of presentation, at $48 \mathrm{~h}$ and 4 weeks later, measuring mediators by nasal absorption and peripheral blood RNA profiling. All patients demonstrated a heightened type 1 immune response. In the first $24 \mathrm{~h}$, patients with asthma had reduced general immune responses, with lower serum IL-6, tumour necrosis factor (TNF)- $\alpha$, CXCL-8, 9, IL-10, IL-17 and CCL-2. Interestingly they demonstrated elevated serum interferon- $\alpha 2 \mathrm{a}$, indicating a more robust type 1 systemic antiviral interferon response, suggesting those with asthma could clearly trigger an interferon response, at least once the immune response to infection has been activated systemically. There was no difference seen in terms of virus titres. Importantly, though, those with asthma did not demonstrate a heightened type 2 immune signature, and although peripheral blood eosinophils and atopic status were not recorded, there was no difference in serum total IgE, periosten, blood eosinophil genes, or nasal IL-13, -4 or -5 , regardless of inhaled or systemic corticosteroid use.

So what do these data tell us? Those with asthma were more likely to present earlier as a consequence of their airway symptoms, as opposed to the seriousness of the systemic influenza infection, as supported by their better clinical outcomes. In all cases, natural severe influenza infection results in a strong, mostly type 1 immune response, with no evidence of heightened type 2 immune responses in asthma, at least acutely. The reduced generalised immune response seen in asthma could be an effect of systemic corticosteroid use, but equally is likely to reflect a less severe systemic illness. An exaggerated systemic immune response was associated with worse outcomes for those without asthma, as has been previously shown [16]. The missing comparator group though is the general public who were otherwise "well" and those with asthma doubtless believed they belonged to, who became infected with influenza but did not need hospitalisation. The susceptibility experienced by those with asthma then appears to rest with their chronic airway disease, not their failure to mount an effective systemic antiviral immune response.

This view is supported by a recent chronic house dust mite model of asthma and influenza infection. The UBIOPRED consortium demonstrated neither an increased type 1 or type 2 response in asthma, but rather an early enhanced innate immune response with heightened release $14 \mathrm{~h}$ after infection of IL-33, KC, TNF- $\alpha$, CXCL-10, IL-12p40 and consequent mucus hypersecretion and airway reactivity, not attenuated by inhaled corticosteroids, but by systemic corticosteroids and a monoclonal antibody to IL-5 [17]. The model could not detect differences in early interferon responses, but probably lacked the precision to see this earliest of innate airway responses. They did see an early increase in viral replication, suggesting the initial antiviral response was, if not impaired, at least delayed, with an exaggerated later systemic interferon- $\gamma$ response. The protection from systemic corticosteroids and anti-IL-5 was associated with evidence of increased airway repair as indicated by enhanced amphiregulin [17]. These results imply the problem lies in the dysregulated response of the airway epithelium and resident immune cells.

The evidence provided by JHA et al. [15] supports the view that the effect of viruses such as influenza on asthma exacerbations is not the result of either exaggerated type 1 or 2 immune responses in asthma, consistent with Salter's view that it is the "exalted sensibility that the inflammation gives rise to" that results in susceptibility to viruses such as influenza. To prevent acute exacerbations in asthma we need to restore or bring balance back to the innate immune responses present in the airways of asthmatics.

Conflict of interest: P.A.B. Wark reports grants from National Health and Medical Research Council, and has participated in clinical trials for Synairgen, outside the submitted work; and has a patent Use of nebulised interferon beta in asthma issued.

\section{References}

1 Salter HH. On Asthma: Its Pathology and Treatment. 1st Edn. London, Churchill, 1860.

2 Johnston SL, Sanderson G, Pattemore PK, et al. Use of polymerase chain reaction for the diagnosis of picornavirus infection in subjects with and without respiratory symptoms. J Clin Microbiol 1993; 31: 111-117. 
3 Jackson DJ, Johnston SL. The role of viruses in acute exacerbations of asthma. J Allergy Clin Immunol 2010; 125: $1178-1187$.

4 Van Kerkhove MD, Vandemaele KA, Shinde V, et al. Risk factors for severe outcomes following 2009 influenza A (H1N1) infection: a global pooled analysis. PLoS Med 2011; 8: e1001053.

5 Jain S, Kamimoto L, Bramley AM, et al. Hospitalized patients with 2009 H1N1 influenza in the United States, April-June 2009. N Engl J Med 2009; 361: 1935-1944.

6 Wark PAB, Johnston SL, Bucchieri F, et al. Asthmatic bronchial epithelial cells have a deficient innate immune response to infection with rhinovirus. J Exp Med 2005; 201: 937-947.

7 Ritchie AI, Jackson DJ, Edwards MR, et al. Airway epithelial orchestration of innate immune function in response to virus infection. A focus on asthma. Ann Am Thorac Soc 2016; 13: Suppl. 1, S55-S63.

8 Bedke N, Sammut D, Green B, et al. Transforming growth factor-beta promotes rhinovirus replication in bronchial epithelial cells by suppressing the innate immune response. PLoS One 2012; 7: e44580.

$9 \mathrm{Wu} \mathrm{Q}$, van Dyk LF, Jiang D, et al. Interleukin-1 receptor-associated kinase M (IRAK-M) promotes human rhinovirus infection in lung epithelial cells via the autophagic pathway. Virology 2013; 446: 199-206.

10 Gill MA, Bajwa G, George TA, et al. Counterregulation between the FceRI pathway and antiviral responses in human plasmacytoid dendritic cells. J Immunol 2010; 184: 5999-6006.

11 Samarasinghe AE, Melo RC, Duan S, et al. Eosinophils promote antiviral immunity in mice infected with influenza A virus. J Immunol 2017; 198: 3214-3226.

12 Ishikawa H, Sasaki H, Fukui T, et al. Mice with asthma are more resistant to influenza virus infection and NK cells activated by the induction of asthma have potentially protective effects. J Clin Immunol 2012; 32: 256-267.

13 Myles P, Nguyen-Van-Tam JS, Semple MG, et al. Differences between asthmatics and nonasthmatics hospitalised with influenza A infection. Eur Respir J 2013; 41: 824-831.

14 Ni Y-N, Chen G, Sun J, et al. The effect of corticosteroids on mortality of patients with influenza pneumonia: a systematic review and meta-analysis. Crit Care 2019; 23: 99.

15 Jha A, Dunning J, Tunstall $\mathrm{T}$, et al. Patterns of systemic and local inflammation in patients with asthma hospitalised with influenza. Eur Respir J 2019; 54: 1900949.

16 Short KR, Kroeze EJBV, Fouchier RAM, et al. Pathogenesis of influenza-induced acute respiratory distress syndrome. Lancet Infect Dis 2014; 14: 57-69.

17 Ravanetti L, Dijkhuis A, Sabogal Pineros YS, et al. An early innate response underlies severe influenza-induced exacerbations of asthma in a novel steroid-insensitive and anti-IL-5-responsive mouse model. Allergy 2017; 72: 737-753. 